\title{
CONTINUED FRACTIONS FOR A CLASS OF TRIANGLE GROUPS
}

\author{
KARIANE CALTA and THOMAS A. SCHMIDT ${ }^{凶}$ \\ (Received 2 April 2011; accepted 1 February 2012; first published online 7 February 2013) \\ Communicated by J. O. Shallit \\ Dedciated to the memory of Alf van der Poorten
}

\begin{abstract}
We give continued fraction algorithms for each conjugacy class of triangle Fuchsian group of signature $(3, n, \infty)$, with $n \geq 4$. In particular, we give an explicit form of the group that is a subgroup of the Hilbert modular group of its trace field and provide an interval map that is piecewise linear fractional, given in terms of group elements. Using natural extensions, we find an ergodic invariant measure for the interval map. We also study Diophantine properties of approximation in terms of the continued fractions and show that these continued fractions are appropriate to obtain transcendence results.
\end{abstract}

2010 Mathematics subject classification: primary 11J70; secondary 11K50, 11J17, 11J81, 20H10. Keywords and phrases: continued fractions, Fuchsian groups, Diophantine approximation.

\section{Introduction}

The celebrated results of Veech [32] highlighting the importance of translation surfaces with large affine diffeomorphism groups have led to various uses of generalizations of regular continued fractions in Teichmüller theory. One aspect has been the determination of explicit algorithms for expansions of the (inverse) slopes of flow directions in terms of parabolic fixed points of a related Fuchsian group, [4, 30, 31].

Veech [32] gave a family of translation surfaces with related Fuchsian triangle groups (of index at most two in a group) of signature $(2, n, \infty)$; the corresponding uniformized hyperbolic surface is of genus zero, has torsion singularities of type 2 and $n$, and has one puncture. Some 40 years earlier Rosen [27] gave continued fraction algorithms for approximation by elements in such triangle groups. The connection between the two is exploited in [5] to show that of Veech's [32] original examples of translation surfaces with nonarithmetic lattice 'Veech' group, precisely those of genus greater than 2 have nonparabolic directions with vanishing Sah-Arnoux-Fathi invariant.

(c) 2013 Australian Mathematical Publishing Association Inc. 1446-7887/2013 \$16.00 
Here we construct continued fraction algorithms for the triangle groups of signature $(3, n, \infty)$ that we show to have various desirable properties. These groups were shown by Ward [33] to also arise from the affine diffeomorphism group of translation surfaces.

For each $n$, Ward rather naturally presents his group with a generating element of order $n$ being the standard rotation of order $n$. However, the Fuchsian group that he gives does not lie in the matrix group with entries only in the algebraic integers. The main properties of Veech groups are conjugation-invariant; we explicitly determine a group that is conjugate to Ward's group, but is contained in the group of matrices of algebraic integer entries, and also has the important property (for applications to translation surfaces) of being in 'standard form' [13]: the extended reals 0,1 and $\infty$ are all cusps for the group.

Having our explicit groups, we then find a rather natural continued fraction map for each $n$, but one that is of infinite invariant measure and fails to enjoy various desirable approximation properties. The infinitude of the measure being due to the existence of a parabolic fixed point for the map in the interval, we define a second algorithm by inducing appropriately with respect to the domain of the corresponding parabolic element. It is this second continued fraction map, $f=f_{n}$, that we then show enjoys desirable properties. In particular, we show that it has no long sequences of poor approximation and that it detects transcendence.

1.1. Main results. For each $n$, let $f(x)=f_{n}(x)$ as given later in Definition 3.11. In a standard manner, to each $x$, our interval map generates a sequence of $f$-approximants, $p_{m} / q_{m}$. We say that $x$ is $f_{n}$-irrational if it has an infinite sequence of approximants. Any such $x$ has its sequence of Diophantine approximation coefficients defined as

$$
\Theta_{m}=\Theta_{m}(x)=q_{m}^{2}\left|x-p_{m} / q_{m}\right| .
$$

Fix $\tau=1+2 \cos \pi / n$. Let $v$ be the probability measure induced on $\mathbb{I}=\mathbb{I}_{n}=[-\tau, 0)$ given as the marginal measure, by integrating $d \mu=(1+x y)^{-2} d x d y$ along the fibers of the region $\Gamma$ defined in Definition 3.13.

Theorem 1.1. For each $n \geq 4$, the following hold.

(i) Every $f$-irrational $x$ is the limit of its $f$-approximants:

$$
\lim _{m \rightarrow \infty}\left|x-p_{m} / q_{m}\right|=0
$$

(ii) For every $f$-irrational $x$ and every $m \geq 1$,

$$
\min \left\{\Theta_{m-1}, \ldots, \Theta_{m+n-1}\right\} \leq \tau,
$$

and the constant $\tau$ is best possible.

(iii) The map $f$ is ergodic with respect to the finite invariant measure $v$ on $\mathbb{I}$.

One test of a usefulness of a continued fraction algorithm is that extremely rapid growth of the denominators $q_{m}$ of the approximants implies transcendence. We show that our continued fractions pass this test. 
THeOREM 1.2. Let $\lambda=2 \cos (\pi / n)$ for any integer $n \geq 4$ and let $d=[\mathbb{Q}(\lambda): \mathbb{Q}]$. If a real number $\xi \notin Q(\lambda)$ is $f$-irrational with convergents $p_{m} / q_{m}$ such that

$$
\limsup _{m \rightarrow \infty} \frac{\log \log q_{m}}{m}>\log (2 d-1)
$$

then $\xi$ is transcendental.

\section{Background}

2.1. Fuchsian groups, translation surfaces, Veech groups. The motivation for developing the continued fraction algorithms in this paper is their usefulness in analyzing the dynamics of the linear flow on certain translation surfaces. A translation surface is a collection of planar polygons glued along parallel sides in such a way that the result is a closed, oriented surface of some genus greater than or equal to one. At the vertices of the polygons, cone points may arise, about which the total angle is $2 \pi n$ for some $n \geq 2$. A first example of a translation surface is a torus that arises from gluing parallel sides of a square, although a torus does not have cone points. It is a theorem of Weyl that on the standard unit torus, in any direction of rational slope, all orbits of the geodesic flow are closed or periodic, while in a direction of irrational slope, all orbits are uniformly distributed. Veech [32] proved that on a certain class of translation surfaces of genus greater than one, now known as the lattice surfaces, the geodesic flow enjoys a simple dynamic dichotomy similar to that of the torus. A lattice surface is one for which the stabilizer of the surface under the natural action of $\mathrm{PSL}_{2}(\mathbb{R})$ is a lattice subgroup of $\mathrm{PSL}_{2}(\mathbb{R})$. Such a group, which is called a Veech group, is also the group of derivatives of the affine diffeomorphisms of the corresponding surface. Since Veech groups are discrete groups of isometries of the hyperbolic plane, they are also Fuchsian groups. Veech also constructed examples of lattice surfaces by gluing together a pair of regular $2 n$-gons, and found the Fuchsian groups mentioned in the Introduction.

Much work has been dedicated to finding new examples of lattice surfaces; we focus on those constructed by Ward [33]. These surfaces arise from reflected copies of a triangle with angles $\pi / 2 n, \pi / n$ and $(2 n-3) \pi / 2 n$ for $n \geq 4$ and their Veech groups are Fuchsian triangle groups of signature $(3, n, \infty)$. In this paper, we create continued fraction algorithms for the Veech groups of the Ward surfaces in the hope of using these algorithms to further study the behavior of the geodesic flow in certain directions on the Ward surfaces.

2.2. Standard number-theoretic planar extension. The notion of natural extension was introduced by Rohlin [26] to aid in the study of ergodic properties of transformations. Since the work of Ito et al. [24], and the initial application of their work by Bosma et al. [9], natural extensions have become an important tool in the study of Diophantine approximation properties of continued fractions as well. For examples of applications to the setting of Rosen continued fractions and the Hecke groups, see, for example, [12, 15, 22, 25]. 
Matrix formulation and approximants. Suppose that $f: J \rightarrow J$ is an interval map that is piecewise fractional linear. Say that $J=\bigcup_{k=1}^{\infty} \Delta_{k}$ with $f(x)=M_{k} \cdot x$ for $x \in J_{k}$, and $M_{k} \in \mathrm{SL}_{2}(\mathbb{R})$ acting projectively. Thus, given general $x$ and $m$,

$$
f^{m}(x)=M_{k_{m}} \cdots M_{k_{2}} M_{k_{1}} \cdot x
$$

and we define

$$
\left(\begin{array}{cc}
q_{m} & -p_{m} \\
-q_{m-1} & p_{m-1}
\end{array}\right):=M_{k_{m}} \cdots M_{k_{2}} M_{k_{1}} .
$$

We define the $m$ th approximant of $x$ as $p_{m} / q_{m}$. Note that

$$
x=\frac{p_{m-1} f^{m}(x)+p_{m}}{q_{m-1} f^{m}(x)+q_{m}} .
$$

Two dimensional map. Let

$$
N_{k}=\left(\begin{array}{rr}
0 & -1 \\
1 & 0
\end{array}\right) M_{k}\left(\begin{array}{rr}
0 & -1 \\
1 & 0
\end{array}\right),
$$

so that $N_{k} \cdot y=-1 /\left(M_{k} \cdot(-1 / y)\right)$. For $x \in \Delta_{k}$, let

$$
\mathcal{T}:(x, y) \mapsto\left(M_{k} \cdot x, N_{k} \cdot y\right) .
$$

Then

$$
\begin{aligned}
\mathcal{T}^{m}(x, 0) & =\left(f^{m}(x), \frac{-1}{M_{k_{m}} \cdots M_{k_{2}} M_{k_{1}} \cdot \infty}\right) \\
& =\left(f^{m}(x), q_{m-1} / q_{m}\right) .
\end{aligned}
$$

Thus, in accordance with, say, [12, p. 1268], we can define the elements of the $\mathcal{T}$-orbit of $(x, 0)$ as

$$
\left(t_{m}, v_{m}\right):=\left(f^{m}(x), q_{m-1} / q_{m}\right) .
$$

Constants of Diophantine approximation. We define the coefficient of Diophantine approximation

$$
\Theta_{m}:=\Theta_{m}(x)=q_{m}^{2}\left|x-p_{m} / q_{m}\right|
$$

From Equation (2.1),

$$
\Theta_{m}=q_{m}^{2}\left|\frac{p_{m-1} f^{m}(x)+p_{m}}{q_{m-1} f^{m}(x)+q_{m}}-\frac{p_{m}}{q_{m}}\right|=\left|\frac{t_{m}}{1+t_{m} v_{m}}\right| .
$$

If we further restrict our matrices $M_{k}$ to be of the form

$$
M_{k}=\left(\begin{array}{rr}
a_{k} & -1 \\
1 & 0
\end{array}\right)
$$

then $t_{m+1}=a_{m}-1 / t_{m}$ and $v_{m+1}=-1 /\left(v_{m}+a_{m}\right)$, and hence also

$$
\Theta_{m}=\left|\frac{v_{m+1}}{1+t_{m+1} v_{m+1}}\right| \text {. }
$$


Natural extension. The natural extension of a system $(f, \mathbb{I}, v, \mathscr{B})$, where $f: \mathbb{I} \rightarrow \mathbb{I}$ is of invariant measure $v$ and $\mathscr{B}$ is the $\sigma$-algebra of Borel sets, is another system $\left(\mathcal{T}, \Omega, \mu, \mathscr{B}^{\prime}\right)$. Here, $T$ is bijective on $\Omega$, and the $\sigma$-algebra $\mathscr{B}^{\prime}$ is generated by the pull-back of $\mathscr{B}$ under a projection $\pi: \Omega \rightarrow \mathbb{I}$.

When $f$ and $\mathcal{T}$ are as above, one has the following key result of [9], deftly reproven (in the classical case) by Jager in [17]. Kraaikamp [19] and Barrionuevo et al. [7] apply Jager's reasoning for other continued fraction type maps.

Proposition 2.1. Suppose that $\left(\mathcal{T}, \Omega, \mu, \mathscr{B}^{\prime}\right)$ is the natural extension of $(f, \mathbb{I}, v, \mathscr{B})$ and that this natural extension is ergodic and given locally as in (2.2), with $d \mu=$ $d x d y /(1+x y)^{2}$, up to normalizing factor. Then, for $v$-almost every $x \in \mathbb{I}$, the sequence $\left(\mathcal{T}^{m}(x, 0)\right)_{m \geq 0}$ is $\mu$-uniformly distributed in $\Omega$.

Jager's proof is so short and attractive that we sketch it here.

Proof [JAGER]. Let $A$ be the set of $x$ such that the orbit of $(x, 0)$ is not $\mu$-uniformly distributed. Let $\mathcal{A}=\{(x, y) \in \Omega \mid x \in A\}$; then for all $(x, y),\left(x, y^{\prime}\right) \in \mathcal{A}$, due to the very definition of $\mathcal{T}$, we have that $\left(\mathcal{T}^{m}(x, y)-\mathcal{T}^{m}\left(x, y^{\prime}\right)\right)$ is a null sequence (that is, has the point $(0,0)$ as its limit). Therefore, for all $(x, y) \in \mathcal{A}$, the orbit of $(x, y)$ is not $\mu$ uniformly distributed. But, the $\mu$ measure of $\mathcal{A}$ is positive if and only if the $v$-measure of $A$ is positive. Since $\mathcal{T}$ is ergodic, we conclude that $v(A)=0$.

\section{Continued fractions for the Ward examples}

3.1. A representative in $\operatorname{PSL}_{2}\left(\boldsymbol{O}_{\boldsymbol{K}}\right)$. For each $n \geq 4$, Ward [33] shows that the translation surface obtained by the unfolding process on the Euclidean triangle of angles $(\pi / 2 n, \pi / n,(2 n-3) \pi / 2 n)$ with $n \geq 4$, has as its Veech group a Fuchsian group of signature $(3, n, \infty)$. For $n>4$, Ward presents his groups with generators

$$
\sigma_{n}=\left(\begin{array}{cc}
1 & \cot \pi /(2 n)+\cot \pi / n \\
0 & 1
\end{array}\right), \quad \beta_{n}=\left(\begin{array}{cc}
\cos \pi / n & \sin \pi / n \\
-\sin \pi / n & \cos \pi / n
\end{array}\right) .
$$

Ward proves that $\sigma_{n}$ and $\beta_{n}$ generate a lattice in $\operatorname{PSL}_{2}(\mathbb{R})$; however, it is easy to see that $\left\langle\sigma_{n}, \beta_{n}\right\rangle$ does not lie in $\operatorname{PSL}_{2}\left(O_{K}\right)$ where $K=\mathbb{Q}(2 \cos (\pi / n))$ is the trace field of the surface. For certain applications of our continued fraction algorithms to translation surfaces, it is desirable to find a conjugate lattice that does lie in $\operatorname{PSL}_{2}\left(O_{K}\right)$. Moreover, we would also like the corresponding conjugated surface to be in standard form, which means that the directions 0,1 and $\infty$ on the surface are algebraically periodic, as defined in [13]; this is guaranteed by 0,1 and $\infty$ being cusps of the group. Calta and Smillie [13] proved that for any translation surface whose Veech group is a lattice and which is in standard form, the set of algebraically periodic directions forms a field that is the trace field of the surface. In this case, one could hope to use number-theoretic results to study the algebraically periodic directions on a lattice surface, in the manner of the recent work of Arnoux and Schmidt for the original Veech examples [5]. 


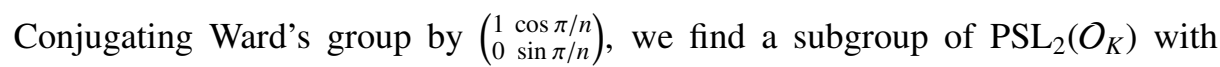
generators

$$
A=\left(\begin{array}{cc}
1 & 1+2 \cos \pi / n \\
0 & 1
\end{array}\right), \quad B=\left(\begin{array}{cc}
2 \cos \pi / n & 1 \\
-1 & 0
\end{array}\right), \quad C=\left(\begin{array}{cc}
1 & -1 \\
1 & 0
\end{array}\right) .
$$

Note that $A B=-C$. Let us denote this group by $\mathcal{W}=\mathcal{W}_{n}=\langle A, B\rangle$. Finally, we check that the conjugated surface is in standard form. First, observe that $\infty$ is clearly a cusp of each $\mathcal{W}_{n}$. And since $B$ sends 0 to $\infty$, it too is a cusp. Also, since $C$ sends $\infty$ to 1,1 must be a cusp as well.

3.2. An initial interval map, but of infinite measure. Fix an $n$ and set $\tau=1+$ $2 \cos \pi / n$. Considering the graph of $C \cdot x$, it is reasonable to let $\mathbb{I}=\mathbb{I}_{n}=[-\tau, 0)$ and define

$$
\begin{aligned}
g: \mathbb{I} & \rightarrow \mathbb{I} \\
x & \mapsto A^{-k} C,
\end{aligned}
$$

where $k=k(x)$ is the unique positive integer such that $g(x) \in \mathbb{I}$. Notice that $g(x)=$ $-k \tau+1-1 / x$. In terms of Section 2.2, we have $M_{k}=A^{-k} C$ and thus the restriction as given in Equation (2.5) holds. The rank-one cylinders

$$
\Delta_{k}=\left[\frac{1}{1-(k-1) \tau}, \frac{1}{1-k \tau}\right),
$$

with $k \geq 2$ are full cylinders; $g$ sends each surjectively onto $\mathbb{I}$. We have $\Delta_{1}=[-\tau, 1 /(1-$ $\tau)$ ), whose image under $f$ is $[-\tau+1+1 / \tau, 0)$. The $g$-orbit of $x=-\tau$ is of importance, thus let

$$
\phi_{j}=g^{j}(-\tau) \quad \text { for } j=0,1, \ldots
$$

We claim that $\phi_{0}, \ldots, \phi_{n-3}$ all lie in $\Delta_{1}$; then $\phi_{n-2} \in \Delta_{2}$; followed by $\phi_{n-1}, \ldots, \phi_{2 n-5}$ back in $\Delta_{1}$; thereafter, $\phi_{2 n-4}=1 /(1-\tau)$ is the left endpoint of $\Delta_{2}$. It follows that $\phi_{2 n-3}=\phi_{0}$. To justify that this is indeed the $g$-orbit of $\phi_{0}$, since $A^{-1} C \cdot x$ is increasing and has no pole in $\mathbb{I}$, it suffices to show the following four things:

$$
\begin{aligned}
\phi_{n-2} & =\left(A^{-1} C\right)^{n-2} \cdot(-\tau)<\left(A^{-2} C\right)^{-1} \cdot 0=1 /(1-2 \tau) ; \\
A^{-2} C\left(\phi_{n-2}\right) & \in \Delta_{1}, \quad\left(A^{-1} C\right)^{n-3} \cdot\left(\phi_{n-1}\right) \in \Delta_{2} \quad \text { and } W \cdot(-\tau)=-\tau,
\end{aligned}
$$

where

$$
W=A^{-2} C\left(A^{-1} C\right)^{n-3} A^{-2} C\left(A^{-1} C\right)^{n-2}=A^{-1} B^{-2} A^{-1} B^{-1}=\left(\begin{array}{cc}
\tau^{2}+1 & \tau^{3} \\
-\tau & -\tau^{2}+1
\end{array}\right) .
$$

These are all easily shown, especially since $\left(A^{-1} C\right)^{n}=B^{n}=\mathrm{Id}$, projectively.

Note that from the above, $\phi_{2 n-4}<\phi_{n-2}$. But then using that $M_{1} \cdot x$ is an increasing function, we easily deduce that the following ordering of real numbers holds:

$$
\phi_{0}<\phi_{n-1}<\phi_{1}<\cdots<\phi_{2 n-4}<\phi_{n-2} .
$$



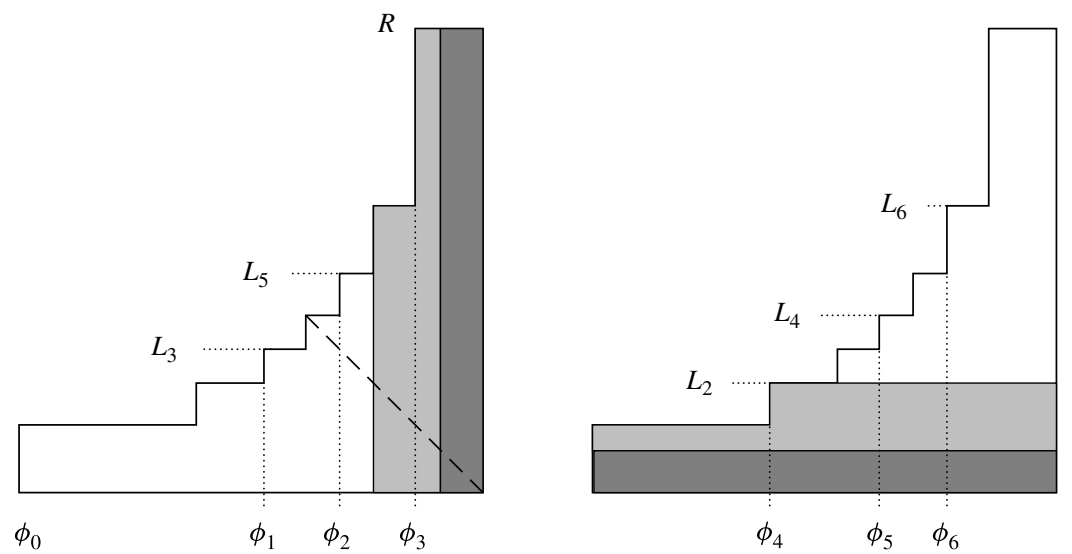

FigURE 1. Natural extension for $n=5$. The region initially lying over $\Delta_{1}$ and its image are shown in white; the region over $\Delta_{2}$ and its image are shown in light gray; and the region above $\Delta_{k}$ with $k>2$ and its image are shown in dark gray. The anti-diagonal $y=-x$ (dashed) is also shown.

Now consider rectangles erected above the $g$-orbit of $\phi_{0}$. Enumerate their heights as $L_{1}, \ldots, L_{2 n-2}$ and $R$, so that the region we are considering (see Figure 1 ) is

$$
\Omega=\bigcup_{i=0}^{n-3}\left[\phi_{i}, \phi_{n-1+i}\right) \times\left[0, L_{2 i+1}\right] \cup \bigcup_{j=1}^{n-2}\left[\phi_{n-2+j}, \phi_{j}\right) \times\left[0, L_{2 i}\right] \cup \bigcup\left[\phi_{n-2}, 0\right) \times[0, R] .
$$

In accordance with the notation of Section 2.2, we have

$$
N_{k}=\left(\begin{array}{cc}
0 & -1 \\
1 & 1-k \tau
\end{array}\right) .
$$

We define $\mathcal{S}(x, y)=\left(M_{k} \cdot x, N_{k} \cdot y\right)$ whenever $x \in \Delta_{k}$.

Proposition 3.1. The map $\mathcal{S}: \Omega \rightarrow \Omega$ is bijective (up to $\mu$-measure zero) when $R=\tau$ and

$$
\begin{gathered}
L_{1}=1 / \tau ; \quad L_{2 i+1}=N_{1} \cdot L_{2 i-1}, \quad 1 \leq i \leq n-3 ; \\
L_{2}=1 /(\tau-1) ; \quad L_{2 j}=N_{1} \cdot L_{2 j-2}, \quad 1 \leq j \leq n-2 .
\end{gathered}
$$

Furthermore, these heights are in increasing order

$$
L_{1}<L_{2}<\cdots<L_{2 n-2}<R
$$

Proof. Direct calculation shows that $N_{k} \cdot \tau=1 /((k-1) \tau-1)$, and $N_{k} \cdot 0=1 /(k \tau-1)$. Therefore $R=\tau$ implies that, for $k \geq 2, \mathcal{S}$ maps $\Delta_{k} \times[0, \tau]$ to

$$
\mathbb{I} \times\left[\frac{1}{k \tau-1}, \frac{1}{(k-1) \tau-1}\right]
$$

and of course this lies directly above the $\mathcal{S}$-image of $\Delta_{k+1} \times[0, \tau]$. 
We have that $\left[\phi_{i}, \phi_{n-1+i}\right) \subset \Delta_{1}$ for $i=0, \ldots, n-4$, and claim that $\mathcal{S}$ sends

$$
\left[\phi_{i}, \phi_{n-1+i}\right) \times\left[0, L_{2 i+1}\right] \quad \text { to } \quad\left[\phi_{i+1}, \phi_{n+i}\right) \times\left[L_{1}, L_{2 i+3}\right] \quad \text { when } i<n-4
$$

and that the image of $\left[\phi_{n-3}, 1 /(1-\tau)\right) \times\left[0, L_{2 n-5}\right]$ is $\left[\phi_{n-2}, 0\right) \times\left[L_{1}, R\right]$. By definition of $N_{1}$, all of this is clear, up to checking that $N_{1}^{n-2} \cdot(1 / \tau)=\tau$. This latter fact follows (depending upon parity of $n$ ) from Lemmas 3.5 and 3.7 below.

Similarly, one finds that $\left[\phi_{n-2+j}, \phi_{j}\right) \times\left[0, L_{2 i}\right]$ is sent by $\mathcal{S}$ to $\left[\phi_{n-1+j}, \phi_{j+1}\right) \times$ $\left[L_{1}, L_{2(i+1)}\right]$ until $\left[\phi_{2 n-4}, \phi_{n-2}\right) \times\left[0, L_{2 n-4}\right]$ is mapped to $\left[\phi_{0}, \phi_{n-1}\right) \times\left[1 /(2 \tau-1), N_{2}\right.$. $\left.L_{2 n-4}\right]$. But

$$
N_{2} \cdot L_{2 n-4}=N_{2} \cdot N_{1}^{n-3} N_{2} N_{1}^{n-2} \cdot L_{1}=L_{1}
$$

follows from our hypotheses and the fact that $W$ fixes $-\tau$. We have thus shown that, up to measure zero, $\mathcal{S}$ is surjective; injectivity is easily verified.

Each of $N_{1} \cdot y$ and $N_{2} \cdot y$ define increasing functions of $y$. Thus, since certainly $1 / \tau<1 /(\tau-1)$, the heights are strictly increasing.

LEMMA 3.2. The region $\Omega$ is of infinite measure.

Proof. Since $\mathcal{S}$ preserves $\mu$, we see that the $\mathcal{S}$-orbit of $\left(\phi_{0}, L_{1}\right)=(-\tau, 1 / \tau)$ lies on the curve $y=-1 / x$. But $d \mu=d x d y /(1+x y)^{2}$ so that $\Omega$ is clearly of infinite $\mu$-measure.

Schweiger [29] formalized a proof in [24] so as to obtain conditions that imply that a planar system is a natural extension of an associated interval map. In particular (see [29, Theorem 21.2.1]), one mainly needs to verify that there is an appropriate algorithm on the second coordinates. It is easily verified that in our setting the map given by $y \mapsto N_{k} \cdot y$ provides that algorithm. (For details pertaining to an application of Schweiger's formalism in a situation that is less straightforward than ours, see [20, Section 4.3].)

Proposition 3.3. With $\Omega$ as in the previous lemma, let $v$ be the measure on I obtained by integration along the fibers. Then $\left(\mathcal{S}, \Omega, \mu, \mathscr{B}^{\prime}\right)$ is the natural extension of $(g, \mathbb{I}, v, \mathscr{B})$, where $\mathscr{B}^{\prime}, \mathscr{B}$ denote the respective $\sigma$-algebra of Borel sets. In particular, the $v$ measure of $\mathbb{I}$ is infinite.

3.3. Bijectivity of the planar map $\mathcal{S}$. Besides giving the calculations that terminate the proof of the bijectivity of $\mathcal{S}$, we also show that the product of the $L_{j}$ with $R=\tau$ equals one. We use an induction proof, relying upon the following elementary result.

Lemma 3.4. Suppose that the $2 \times 2$ real matrix $M$ is of determinant one and has the form $M=\left(\begin{array}{rr}a & b \\ -b & 0\end{array}\right)$. Then for any real $x$,

$$
M \cdot x=\frac{1}{M^{-1} \cdot(1 / x)} .
$$

Proof. This is immediately verified. 
We also note the following useful formula. Using the conjugation expressing $B$ in terms of $\beta_{n}$ given in Section 3.1, one easily verifies that, for any integer $j$,

$$
B^{j}=\frac{1}{\sin (\pi / n)}\left(\begin{array}{cc}
\sin \frac{(j+1) \pi}{n} & \sin \frac{j \pi}{n} \\
-\sin \frac{j \pi}{n} & -\sin \frac{(j-1) \pi}{n}
\end{array}\right) .
$$

Lemma 3.5. Let $n$ be even. Then, with notation as above, $\phi_{n / 2-1}=-1$. Furthermore, $N_{1}^{n-2} \cdot(1 / \tau)=\tau$.

Proof. First note that $\phi_{n / 2-1}=\left(A^{-1} C\right)^{n / 2-1} \cdot(-\tau)$, by the calculation of the orbit of $\phi_{0}$ above. It suffices to show that $B^{n / 2-1} \cdot(-\tau)=-1$, or that $B^{n / 2} \cdot(-\tau)=B \cdot(-1)$, since $B=A^{-1} C$. Direct calculation shows that $B \cdot(-1)=-\tau+2$. From (3.2),

$$
B^{n / 2}=\left(\begin{array}{cc}
-\cot \pi / n & -\csc \pi / n \\
\csc \pi / n & \cot \pi / n
\end{array}\right),
$$

and direct calculation shows that $B^{n / 2} \cdot(-\tau)=-\tau+2=B \cdot(-1)$. Finally, one easily verifies that $B^{n / 2} \cdot(2-\tau)=-1 / \tau$, and $N_{1}^{n-2} \cdot(1 / \tau)=\tau$ follows.

Corollary 3.6. Let $n$ be even. Then for $0 \leq j \leq n / 2-1$,

$$
\phi_{n / 2-1-j} \phi_{n / 2-1+j}=1 \text {. }
$$

Furthermore, the product $R \cdot \prod_{j=1}^{2 n-4} L_{j}=1$.

Proof. The displayed equation obviously holds when $j=0$. We use induction, repeatedly applying Lemma 3.4 with $M=M_{1}=A^{-1} C$ for $1 \leq j \leq n / 2-1$. Thereafter we apply this lemma with $M=M_{2}=A^{-2} C$, and then again with a series of $M=A^{-1} C$.

Now, we have that the various heights $R, L_{j}$ are $y$-coordinates of 'corner' points whose corresponding $x$-coordinates are in the orbit of $\phi_{0}$. Since these corner points lie on the curve $y=1 / x$, the result follows.

Lemma 3.7. Let $n=2 m+3$. Then $\left(A^{-1} C\right)^{m} A^{-2} C\left(A^{-1} C\right)^{n-2}(-\tau)=-1$ and so $\phi_{3 m+2}=$ -1 . Furthermore, $N_{1}^{n-2} \cdot(1 / \tau)=\tau$.

Proof. We have that $B^{n-2}=B^{-2}$, but

$$
B^{-2}=\left(\begin{array}{cc}
-1 & 1-\tau \\
\tau-1 & (1-\tau)^{2}-1
\end{array}\right),
$$

giving $B^{n-2} \cdot(-\tau)=B^{-2} \cdot(-\tau)=-1 / \tau$. From this, $N_{1}^{n-2} \cdot(1 / \tau)=\tau$ follows.

Now

$$
A^{-2} C=\left(\begin{array}{cr}
1-2 \tau & -1 \\
1 & 0
\end{array}\right)
$$

and so $A^{-2} C \cdot(-1 / \tau)=1-\tau$. 
It remains to show that $B^{m} \cdot(1-\tau)=-1$. By (3.2),

$$
B^{m} \cdot(-2 \cos (\pi / n))=\frac{-2 \cos \frac{\pi}{n} \sin \frac{(m+1) \pi}{n}+\sin \frac{m \pi}{n}}{2 \cos \frac{\pi}{n} \sin \frac{m \pi}{n}-\sin \frac{(m-1) \pi}{n}} .
$$

Elementary trigonometric manipulations show that the numerator is $-\sin ((m+2) \pi / n)$. Similarly, the denominator has value $\sin ((m+1) \pi / n)$. Since $m=(n-3) / 2$, we are evaluating the quotient of $-\sin ((n+1) \pi / 2 n)$ by $\sin ((n-1) \pi / 2 n)$. We thus find that $B^{m} \cdot(1-\tau)=-1$. The rest follows directly.

Applying Lemma 3.4 as in the proof of Lemma 3.6 now allows one to show the following.

Corollary 3.8. Let $n=2 m+3$ be odd. Then for $0 \leq j \leq m$, one has both

$$
\phi_{3 m+2-j} \phi_{3 m+2+j}=1 \text { and } \phi_{j} \phi_{n-2+j}=1 .
$$

Furthermore, the product $R \cdot \prod_{j=1}^{2 n-4} L_{j}=1$.

3.4. Interval map, $f$. The infinitude of the measure of I can be seen as caused by the fact that the parabolic $W$, as given in Equation (3.1), fixes $\phi_{0}=-\tau$. We thus 'accelerate' the map $g(x)$, by inducing past $W^{-1} \cdot[-\tau, 0)$, and thereby find our interval map $f(x)$.

Definition 3.9. Let $\varepsilon_{0}=W^{-1} \cdot 0$, that is, $\varepsilon_{0}=-\tau^{3} / 1+\tau^{2}$.

Lemma 3.10. For $x \in\left(-\tau, \varepsilon_{0}\right)$, let $j(x)$ be minimal such that $g^{j(x)}(x)>\varepsilon_{0}$. Then

$$
j(x)=-1+\left\lceil\frac{-1}{\tau^{2}}+\frac{1}{\tau(\tau+x)}\right\rceil .
$$

Proof. Conjugating by the map $x \mapsto x+\tau$ sends $W$ to $\left(\begin{array}{cc}1 & 0 \\ -\tau & 1\end{array}\right)$. From this one solves to find $j(x)$.

Definition 3.11. Let $f: \mathbb{I} \rightarrow \mathbb{I}$ be given by

$$
f(x)= \begin{cases}g(x) & \text { if } x>\varepsilon_{0} \\ g^{j(x)}(x) & \text { otherwise }\end{cases}
$$

Lemma 3.12. Let $\varepsilon_{i}:=f^{i}\left(\varepsilon_{0}\right)$. Then

$$
\phi_{0}<\varepsilon_{0}<\phi_{n-1}
$$

and, for $\ell=1, \ldots, n-3$,

$$
\phi_{\ell}<\varepsilon_{\ell}<\varepsilon_{n-2+\ell}<\phi_{n-1+\ell} .
$$

Furthermore, $\varepsilon_{2 n-4}=1 /(1-2 \tau)$. 

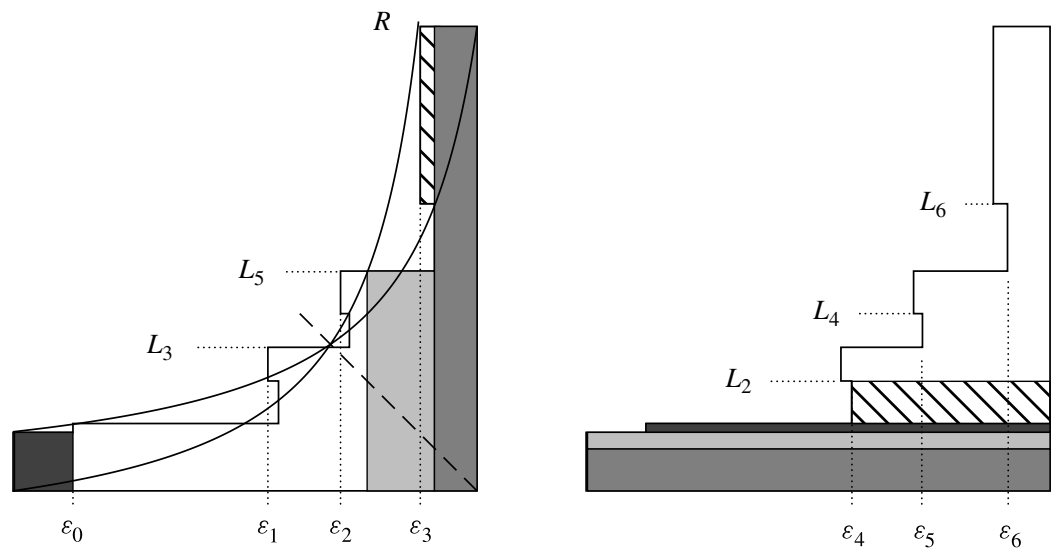

FigURE 2. Natural extension of domain $\Gamma$, for accelerated map, $n=5$. The region initially lying over the accelerated subinterval is shaded darkest; the remainder lying above $\Delta_{1}$ is shown in white; the lower region over $\Delta_{2}$ is shown in light gray; the high region over (part of) $\Delta_{2}$ is shown lined; and the region above $\Delta_{k}$ with $k>2$ is shown in medium gray. Likewise their images. Curves corresponding to $\theta(x, y)=\tau, y=-x$ (dashed) and related to $\theta\left(\mathcal{T}^{-1}(x, y)\right)=\tau$ are also shown.

Proof. Since $L_{2}=1 /(\tau-1)$, it follows that $\phi_{n-1}=1-\tau$. One then trivially verifies that $-\tau<\varepsilon_{0}<\phi_{n-1}$ holds, since $2<\tau=1+2 \cos \pi / n<3$.

The point $\alpha_{1}:=M_{2}^{-1} \cdot 0=1 /(1-2 \tau)$ is in $M_{1} \cdot \Delta_{1}$, and further $\phi_{2 n-4}<\phi_{n-2}<\alpha_{1}$. Thus writing $\alpha_{i}=g^{-1}\left(\alpha_{1}\right)$, we find that $\phi_{2 n-4-j}<\phi_{n-2-j}<\alpha_{j+1}$ for $j=0, \ldots, n-3$. Furthermore, $\alpha_{j+1}<\phi_{2 n-3-j}$ for $j=1, \ldots, n-3$, since $M_{1} \cdot \phi_{2 n-4}=0$. One completes this backwards orbit so as to find that $\alpha_{2 n-3}$ must equal $\varepsilon_{0}$. In particular, $\varepsilon_{2 n-4}=$ $1 /(1-2 \tau)$. But also it follows that $\phi_{n-2}<\varepsilon_{n-2}<\varepsilon_{2 n-4}$, from which the remaining inequalities follow.

We now define a new region (see Figure 2).

Definition 3.13. Let

$$
\begin{aligned}
\Gamma=[-\tau, & \left.\varepsilon_{0}\right) \times\left[0, \tau /\left(\tau^{2}+1\right)\right] \cup\left[\varepsilon_{0}, \varepsilon_{1}\right) \times\left[0, L_{1}\right] \\
& \cup \bigcup_{j=1}^{n-3}\left(\left[\varepsilon_{j}, \varepsilon_{n-2+j}\right) \times\left(\left[0, L_{j}\right] \cup\left[L_{2 j}, L_{2 j+1}\right]\right) \cup\left[\varepsilon_{n-2+j}, \varepsilon_{j+1}\right) \times\left[0, L_{2 j+1}\right]\right) \\
& \cup\left[\varepsilon_{n-2}, \frac{1}{1-2 \tau}\right) \times\left(\left[0, L_{2 n-5}\right] \cup\left[L_{2 n-4}, \tau\right]\right) \cup\left[\frac{1}{1-2 \tau}, 0\right) \times[0, \tau] .
\end{aligned}
$$

In order to define cylinder sets for $g(x)$ indicated by a single subscripting index, we now define $\Delta_{k}^{\prime}$ for $k$ nonzero integers.

Definition 3.14. If $k>2$, then let $\Delta_{k}^{\prime}=\Delta_{k}$. Further, let $\Delta_{1}^{\prime}=\left[\varepsilon_{0}, 1 /(1-\tau)\right)$ and

$$
\Delta_{j}^{\prime}=\left[-\tau+\frac{\tau}{-(j-1) \tau^{2}+1},-\tau+\frac{\tau}{-j \tau^{2}+1}\right)
$$

for $j \leq-1$. 
In particular, for $x \in \Delta_{j}^{\prime}$ with $j \leq-1$, we have $g(x)=W^{-j} \cdot x$. Accordingly, let $M_{-j}=W^{j}$ and $N_{-j}$ be its conjugate by $\left(\begin{array}{ll}0 & -1 \\ 1 & 0\end{array}\right)$. Thus, we have defined cylinder sets of index in $-\mathbb{N} \cup \mathbb{N}$, and correspondingly indexed maps.

For brevity, when we write that a two-dimensional map is the natural extension of an interval map we mean that a relationship holds analogously to the formal statement included in Proposition 3.3.

Proposition 3.15. Define $\mathcal{T}: \Gamma \rightarrow \Gamma$ by $\mathcal{T}(x, y)=\left(M_{k} \cdot x, N_{k} \cdot y\right)$ whenever $x \in \Delta_{k}^{\prime}$ for $k \in-\mathbb{N} \cup \mathbb{N}$. Then:

(i) $\mathcal{T}$ is a bijection up to $\mu$-measure zero;

(ii) $\Gamma$ is a finite measure subset of $\Omega$;

(iii) the map $\mathcal{T}: \Gamma \rightarrow \Gamma$ gives a natural extension of $f: \mathbb{I} \rightarrow \mathbb{I}$.

Proof. By definition, $\mathcal{T}$ agrees with $\mathcal{S}$ on $[1 /(1-2 \tau), 0) \times[0, \tau]$, giving an image of $[-\tau, 0) \times(0,1 /(2 \tau-1)]$. Also on $\left[\varepsilon_{n-2}, 1 /(1-2 \tau)\right) \times\left[L_{2 n-4}, \tau\right]$, the maps $\mathcal{T}$ and $\mathcal{S}$ agree, and give as image $\left[\varepsilon_{n-1}, 0\right) \times\left[L_{1}, L_{2}\right]$.

From Lemma 3.12, one has that $\varepsilon_{2 n-2}<1 /(1-\tau)$; therefore, $\Gamma$ includes the region

$$
\left[\frac{1}{1-\tau}, \frac{1}{1-2 \tau}\right) \times\left[0, L_{2 n-5}\right]
$$

upon which $\mathcal{T}$ agrees with $\mathcal{S}$, and gives an image of $[-\tau, 0) \times\left[1 /(2 \tau-1), N_{2} \cdot L_{2 n-5}\right]$. But, by Proposition 3.1, one has

$$
N_{2} \cdot L_{2 n-5}=N_{2} N_{1}^{-1} \cdot \tau=\frac{\tau}{\tau^{2}+1}
$$

A calculation confirms that $N_{-j^{\prime}}$ sends

$$
\left[0, \frac{\tau}{\tau^{2}+1}\right] \text { to }\left[\frac{j^{\prime} \tau}{j^{\prime} \tau^{2}+1}, \frac{\left(1+j^{\prime}\right) \tau}{\left(1+j^{\prime}\right) \tau^{2}+1}\right] .
$$

By definition, $W^{j^{\prime}} \cdot \Delta_{-j^{\prime}}^{\prime}=\left[\varepsilon_{0}, 0\right)$, and thus we find that $\mathcal{T}$ sends

$$
\left[0, \varepsilon_{0}\right) \times\left[0, \frac{\tau}{\tau^{2}+1}\right] \text { to }\left[\varepsilon_{0}, 0\right) \times\left[\frac{\tau}{\tau^{2}+1}, L_{1}\right] \text {. }
$$

On the remaining portion of $\Gamma$, one has agreement of $\mathcal{T}$ with $\mathcal{S}$, and the image is easily calculated. In particular, one finds that $\mathcal{T}$ is bijective up to measure zero, and that $\Gamma$ is a closed proper subset of $\Omega$. Since $\tau /\left(\tau^{2}+1\right)<L_{1}$, we have that $\left(\phi_{0}, L_{1}\right) \notin \Gamma$. It follows that the $\mathcal{S}$-orbit of this point is not in $\Gamma$, and from this one easily finds that $\Gamma$ is bounded away from the curve $y=-1 / x$. Therefore, $\Gamma$ is of finite $\mu$-measure. Finally, one checks that $\mathcal{T}$ is the induction to $\Gamma$ of $\mathcal{S}$ just as $f$ is induced from $g$, and thus Proposition 3.3 implies that $\mathcal{T}$ gives the natural extension of $f$.

\section{Ergodicity and Diophantine properties from natural extensions}

We use the system $(\mathcal{T}, \Gamma, \mu)$, where $\mu$ denotes the probability measure on $\Gamma$ induced by $d \mu=(1+x y)^{-2} d x d y$, to find egodicity as well as to study Diophantine properties of the interval maps $f_{n}$. 
4.1. Convergence. Consecutive Diophantine approximation constants have an easily computed ratio, when $\mathcal{T}$ is locally of a particularly nice form.

Lemma 4.1. Consider the function $h$ given by

$$
h:(x, y) \mapsto(M \cdot x, N \cdot y),
$$

with $M \cdot x=a+\varepsilon / x, \quad N \cdot y=1 /(-a+\varepsilon y))$, and $\varepsilon= \pm 1$. Then

$$
\theta(h(x, y)) / \theta(x, y)=-(M \cdot x) /(N \cdot y)
$$

Proof. This is a matter of direct calculation.

Proposition 4.2. For any $x$ of infinite $f$-orbit

$$
\lim _{m \rightarrow \infty}\left|x-p_{m} / q_{m}\right|=0
$$

where $p_{m} / q_{m}$ are the approximants of $x$ as in (2.1).

Proof. By definition, $\left|x-p_{m} / q_{m}\right|=\Theta_{m} / q_{m}^{2}$. The ratio of two consecutive values is then

$$
\frac{\Theta_{m} / q_{m}^{2}}{\Theta_{m-1} / q_{m-1}^{2}}=v_{m}^{2} \frac{\Theta_{m}}{\Theta_{m-1}},
$$

since $q_{m}=q_{m-1} / v_{m}$, using the notation of (2.3). Thus, if $\left(t_{m}, v_{m}\right)=\left(M \cdot t_{m-1}, N \cdot v_{m-1}\right)$ with $M, N$ meeting the hypotheses of Lemma 4.1, then this ratio is $-t_{m} v_{m}$. Since statement (ii) of Proposition 3.15 gives that the region $\Gamma$ is bounded away from $y=-1 / x$, there is a $0<\delta<1$ such that the ratio in question is between zero and $\delta$.

Now, the only setting in which $\mathcal{T}(x, y)=(M \cdot x, N \cdot y)$ with $M, N$ not of the form of Lemma 4.1 is where there is some $j$ such that $M=W^{j}$. But we have $W^{j}=$ $\left(M_{2} M_{1}^{n-3} M_{2} M_{1}^{n-2}\right)^{j}$, thus we can apply Lemma 4.1 to the various intermediate steps, and since the original domain $\Omega$ is bounded above by the curve $y=-1 / x$, we are assured that if $M=W^{j}$, then the ratio $\left(\Theta_{m} / q_{m}^{2}\right) /\left(\Theta_{m-1} / q_{m-1}^{2}\right)$ is bounded above by 1 .

Since applications of $W^{j}$ are isolated, the convergence to zero of the geometric sequence of ratio $\delta$ implies that $\lim _{m \rightarrow \infty}\left|x-p_{m} / q_{m}\right|=0$.

REMARK 4.3. A traditional way to show convergence of the approximants of a continued fraction algorithm is to show that the coefficients of approximation, the $\Theta_{m}$, are bounded (which is indeed the case here), and then to show that the denominators of the approximants, the $q_{m}$, are strictly increasing and grow to infinity. Here, the $q_{m}$ are not strictly increasing, as is shown immediately by the fact that $q_{m-1} / q_{m}=v_{m}$ is a $y$-coordinate in $\Gamma$, and thus can be greater than 1 .

Note that our approach is also viable for other continued fraction maps, such as the well-studied Rosen continued fractions. 
4.2. Ergodicity. In this section we apply arguments similar to those of [12] for the Rosen $\lambda$-continued fractions to prove the following result.

Theorem 4.4. Both the system $(f, \mathbb{I}, v, \mathscr{B})$ and its natural extension $\left(\mathcal{T}, \Gamma, \mu, \mathscr{B}^{\prime}\right)$ are ergodic.

A system and its natural extension are jointly either ergodic or not, and a system is ergodic if any reasonable (see [29, Theorem 17.2.4]) induced system is ergodic. Since the weak Bernoulli property implies ergodicity, we show that a system induced from $f$ is weak Bernoulli.

Recall that if $(I, f, \rho, \mathcal{P})$ is a dynamical process for which $f$ acts on $I, \rho$ is an invariant probability measure on $I$ and $\mathcal{P}$ is a partition of the system, then $(I, f, \rho, \mathcal{P})$ is said to be weak Bernoulli if the sequence $\beta_{n} \rightarrow 0$ where

$\beta_{n}=\sup _{L \geq 1}\left\{\sum_{i, j}\left|\rho\left(A_{i} \cap T^{-n-L}\left(A_{j}\right)\right)-\rho\left(A_{i}\right) \rho\left(A_{j}\right)\right|:\left\{A_{i}\right\}\right.$ is the set of cylinders of rank $\left.L\right\}$.

For fixed $\tau=1+2 \cos (\pi / n)$, let $\mathbb{I}=\mathbb{I}_{n}, f=f_{n}, \rho=\rho_{n}$ and $\mathcal{P}$ be the partition given by the cylinders of $f_{n}$. Given an $m$-tuple $\left(a_{1}, \ldots, a_{m}\right)$ of nonzero integers, we let $\left[a_{1}, \ldots, a_{m}\right]=\bigcap_{j=1}^{n} f^{-j+1}\left(\Delta_{a_{j}}^{\prime}\right)$ and say that the $m$-tuple is admissible if $\left[a_{1}, \ldots, a_{m}\right]$ is a rank $m$ cylinder for $f$ in the sense that the intersection in this definition has positive $\rho$-measure. Compare the following with Figure 2. The restrictions on the $a_{i}$ are: (1) a negative integer $a_{i}$ can only be preceded by $a_{i-1}>1$; (2) there are at most $n-2$ consecutive $a_{i}=1$; (3) $n-2$ consecutive 1 s succeeded by a 2 can be followed by at most $n-3$ consecutive $1 \mathrm{~s}$; (4) a sequence realizing the maximum in the previous restriction can only be succeeded by a 3 or greater.

To show that $(I, f, \rho, \mathcal{P})$ is ergodic, we show that a particular induced process is weak Bernoulli and thus a fortiori ergodic. Let $Y$ be the union of the rank-one cylinders for digits at least 3 for $f$, that is, $Y=\bigcup_{n=3}^{\infty} \Delta_{n}=[1 /(1-2 \tau), 0)$. Let $f_{Y}$ be the induced transformation on $Y$, that is, $f_{Y}(y)=f^{m}(y)$ where $m=\inf \left\{k>0: f^{k}(y) \in Y\right\}$. Furthermore, let $\rho_{Y}$ be the normalized probability measure on $Y$. Also let $Q$ be the partition of the system $\left(Y, f_{y}, \rho_{Y}\right)$ given by the sets

$$
Q_{\alpha}=\left[a_{1}, a_{2}, \ldots, a_{m}\right] \cap f^{-m}(Y)
$$

where $\alpha=\left(a_{1}, \ldots a_{m}\right)$ and each index $\alpha=\left(a_{1}, \ldots, a_{m}\right)$ is admissible, $a_{1} \geq 3, a_{i}<3$ for $i>2$. Note that this last condition simply guarantees that if $\alpha=\left(a_{1}, \ldots, a_{m}\right)$, then for any $y \in Q_{\alpha}, f^{k}(y) \notin Y$ for any $k<m$.

To prove that the induced process $\left(Y, f_{Y}, \rho_{Y}, Q\right)$ is weak Bernoulli, we show that it satisfies Adler's criteria:

(i) $f_{Y}$ maps $Q_{\alpha}$ onto $Y$ for each $\alpha$;

(ii) $f_{Y}$ restricted to each $Q_{\alpha}$ is twice differentiable;

(iii) $\inf _{x \in Y}\left|f_{Y}^{\prime}(x)\right|>1$;

(iv) $\sup _{\alpha} \sup _{x, y \in Q_{\alpha}}\left|f_{Y}^{\prime \prime}(x)\right| /\left|f_{Y}^{\prime}(y)\right|^{2}<\infty$. 
Proposition 4.5. The process $\left(Y, f_{Y}, \rho_{Y}, Q\right)$ is weak Bernoulli.

Proof. That criterion (i) is satisfied follows from the facts that all rank-one cylinders $\Delta_{a}^{\prime}$ for $a>1$ are full and that $\varepsilon_{2 n-4}=1 /(1-2 \tau)$. (Note that if we had included $\Delta_{2}$ in our $Y$, then this criterion would not be satisfied.)

Criterion (ii) follows from the fact that if $\alpha=\left(a_{1}, \ldots, a_{m}\right)$, then on $Q_{\alpha}, f_{Y}=f^{m}$, and this is certainly twice differentiable.

To see that criterion (iii) is satisfied, note that if $x \in Q_{\alpha}$ for $\alpha=\left(a_{1}, \ldots, a_{m}\right)$, then $f_{Y}(x)=f^{m}(x)$. The chain rule allows us to prove that the derivative of $f^{m}(x)$ is greater than one in absolute value, by showing that $\left|f^{\prime}\left(f^{m-1}(x)\right) \cdots f^{\prime}(x)\right|>1$.

Now, if $k \geq 1$ and $x \in \Delta_{k}$, then $f(x)=A^{-k} C \cdot x=1-k \tau-1 / x$. Thus $f^{\prime}(x)=1 / x^{2}$ and $\left|f^{\prime}(x)\right|>1$ exactly when $x \in(-1,0)$.

If $x \in\left[-\tau, \varepsilon_{0}\right)$, the accelerated interval upon which powers of the matrix $W$ are applied, then using Equation (3.1) we easily have that $\left|f^{\prime}(x)\right| \geq 1$. It thus remains to examine the interval $\left[\epsilon_{0},-1\right)$, upon which $M:=M_{1}=A^{-1} C$ is applied. Suppose that $x \in\left[\epsilon_{0},-1\right)$ and $M^{j} \cdot x \in[1 /(1-2 \tau), 0)=Y$. Then certainly $x \in\left[M^{-(j+1)} \cdot 0, M^{-j} \cdot 0\right]$. For ease of reading, write $M(x)=M \cdot x$ and similarly for any function defined by a matrix action, so that we wish to show that $\left|\left(M^{j}\right)^{\prime}(x)\right|>1$. Now $\left(M^{j}\right)^{\prime}(x)=1 / d(x)^{2}$ where

$$
d(x)=M^{j}(x) M^{j-1}(x) \cdots x .
$$

Thus it suffices to show that $|d(x)|<1$. To do this, we show that $d(x)$ vanishes on the right endpoint of the interval, that $|d(x)|<1$ at the left endpoint and that $d(x)$ is appropriately increasing or decreasing on the entire interval.

First note that $d\left(M^{-j}(0)\right)=0$ because of the leftmost factor of $d(x)$ and that

$$
d\left(M^{-j+1}(0)\right)=M^{-j+1}(0) M^{-j}(0) \cdots M^{-1}(0) .
$$

One can show inductively that $M^{-j}(0)=-B_{j} / B_{j+1}$ where the sequence $B_{j}$ is defined recursively as $B_{0}=0, B_{1}=1$ and $B_{k+1}=\lambda B_{k}-B_{k-1}$. Thus

$$
d\left(M^{-j}(0)\right)=\left(-B_{1} / B_{2}\right)\left(-B_{2} / B_{3}\right) \cdots\left(-B_{j} / B_{j+1}\right),
$$

which simplifies as $(-1)^{j} / B_{j+1}$. Now, as reported in [12], $B_{k}=\sin (k \pi / n) / \sin (\pi / n)$, which is in particular greater than one in absolute value. Thus $\left|d\left(M^{-(j+1)}(0)\right)\right|<1$ for all $j$.

We now show that if $j$ is even then $d(x)$ decreases on $\left[M^{-(j+1)}(0), M^{-j}(0)\right]$, but that $d(x)$ increases if $j$ is odd. Using the product rule,

$$
d^{\prime}(x)=\prod_{i=1}^{j} M^{i}(x)+\sum_{k} x D_{k}(x)
$$

where $D_{k}(x)$ is the derivative of $M^{k}(x)$ times the product indexed with $i \neq k$ of the $M^{i}(x)$. If $j$ is even, the first term in the sum is positive because each $M^{i}(x)<0$; also each summand $x D_{k}(x)$ is positive because $x$ is negative, $\left(M^{k}(x)\right)^{\prime}$ is positive 
and the product of the rest of the terms is negative. Thus if $j$ is even, $d^{\prime}(x)>0$ on $\left[M^{-(j+1)}(0), M^{-j}(0)\right]$; and similarly, if $j$ is odd, $d^{\prime}(x)<0$. And in either case, we find that $|d(x)|<1$ on the entire interval. Thus $\left|f_{Y}^{\prime}(x)\right|>1$ for all $x \in Y$.

We now turn to criterion (iv). Elementary considerations show that the supremum of $\left|f_{Y}^{\prime \prime}(x)\right| /\left|f_{Y}^{\prime}(y)\right|^{2}$ is bounded for $x, y \in Q_{\alpha}$ over all $\alpha=\left(a_{1}\right)$. We will thus suppose in what follows that $\alpha=\left(a_{1}, \ldots, a_{m}\right)$ with $m>1$. With $x \in Q_{\alpha}$, from $f_{Y}(x)=f^{m}(x)$, we have that

$$
f_{Y}(x)=\frac{q_{m} x-p_{m}}{p_{m-1}-x q_{m-1}} .
$$

If we also suppose that $y \in Q_{\alpha}$, then since all of $p_{j}, q_{j}$ for $j=0, \ldots, m$ are common to $x$ and $y$, from the above equality we see that

$$
\left|f_{Y}^{\prime \prime}(x)\right| /\left|f_{Y}^{\prime}(y)\right|^{2}=2 q_{m-1}\left(q_{m-1} y-p_{m-1}\right)^{4} /\left|q_{m-1} x-p_{m-1}\right|^{3}
$$

and it is this quantity we must bound. Recalling that $\Theta_{m}(x)=q_{m}^{2}\left|x-p_{m} / q_{m}\right|$, we can rewrite this as

$$
2 \Theta_{m-1}(y)\left[\Theta_{m-1}(y) / \Theta_{m-1}(x)\right]^{3} .
$$

Now, there is a finite upper bound (depending only on $\Gamma$ ) for $\Theta_{m-1}(x), \Theta_{m-1}(y)$, as one easily sees from (2.4) and statement (ii) of Proposition 3.15; in the $(t, v)$-plane in our region $\Gamma$ is bounded away from $t v=-1$. By elementary considerations of partial derivatives, we find a (positive) lower bound on $\Theta_{m-1}(x)=-t_{m-1} /\left(1+t_{m-1} v_{m-1}\right)$ by letting $v_{m-1}$ take its lower bound value, zero; and letting $t_{m-1}=f^{m-1}(x)$ take its upper bound value in this setting, $1 /(1-2 \tau)$. We conclude that there is a global finite upper bound on $\left|f_{Y}^{\prime \prime}(x)\right| /\left|f_{Y}^{\prime}(y)\right|^{2}$.

4.3. No long sequences of poor approximation. In this subsection, we prove the following result, similar to results of Borel in [8], which excludes long sequences of poor approximation by $p_{m} / q_{m}$ to $x$. Recall that Borel showed that for every irrational number $x$, and every $n \geq 1$ with the classical constants of approximation $\theta_{k}$,

$$
\min \left\{\theta_{n-1}, \theta_{n}, \theta_{n+1}\right\}<\frac{1}{\sqrt{5}} .
$$

The constant $1 / \sqrt{5}$ is best possible. Our approach is related to that of [22]. Recall that $\tau=1+2 \cos \pi / n$.

Proposition 4.6. For every $f$-irrational $x$ and every $m \geq 1$,

$$
\min \left\{\Theta_{m-1}, \ldots, \Theta_{m+n-1}\right\} \leq \tau
$$

and the constant $\tau$ is best possible.

Since poor approximation is signaled by large approximation coefficients, we define the following. 
DeFinition 4.7. Let

$$
\theta(x, y)=\frac{-x}{1+x y},
$$

and define the region of large coefficients of Diophantine approximation to be

$$
\mathcal{D}=\left\{P \in \Gamma \mid \theta(P)>\tau, \theta\left(\mathcal{T}^{-1}(P)\right)>\tau\right\}
$$

We have the following immediate result.

Lemma 4.8. For $x \in \mathbb{I}_{n}$ and $m \in \mathbb{N}, \min \left\{\Theta_{m-1}(x), \Theta_{m}(x)\right\}>\tau$ if and only if $\mathcal{T}^{m}(x, 0) \in \mathcal{D}$.

Proof. Equation (2.4) shows that, for $x \in \mathbb{I}_{n}$,

$$
\theta\left(\mathcal{T}^{m}(x, 0)\right)=\theta\left(t_{m}, v_{m}\right)=\Theta_{m}(x) .
$$

Thus the result holds.

Only a small number of consecutive elements of a $\mathcal{T}$-orbit can lie in the region $\mathcal{D}$.

Proposition 4.9. Given any $P \in \Omega$, at least one of $\left\{P, \mathcal{T}(P), \ldots, \mathcal{T}^{n-2}(P)\right\}$ lies outside of $\mathcal{D}$. Furthermore, there exist points $Q$ such that $\left\{Q, \mathcal{T}(Q), \ldots, \mathcal{T}^{n-3}(Q)\right\} \subset \mathcal{D}$.

Proof. The first result holds as soon as we show that the projection to the first coordinate sends $\mathcal{D}$ to a subset of $\Delta_{1}^{\prime}$, for the $f$-orbit of any $x$ can lie in $\Delta_{1}^{\prime}$ at most $n-3$ consecutive times.

We consider the location relative to the boundary of $\mathcal{D}$ of some corner points of $\Omega$. By Equations (2.4) and (2.6), a point $P=(x, y)$ whose pre-image is not of $x$ coordinate in $\left[-\tau, \varepsilon_{0}\right)$ lies in $\mathcal{D}$ if and only if both $y>-1 / x-1 / \tau$ and $y>\tau /(1-\tau x)$. First note that $(-\tau, 0)$ and $(0, \tau)$ lie on the curves that restrict to give the boundary. Now, since $L_{2 n-5}=N_{1}^{-1} \cdot \tau$, it follows that $P_{\infty}:=\left(1 /(1-\tau), L_{2 n-5}\right)$ (when $n=5$, this is the top left corner 'light gray' point of the left region of Figure 2) lies on the curve of equation $y=-1 / x-1 / \tau$. Since the map $(x, y) \mapsto\left(M_{2} \cdot x, N_{2} \cdot y\right)$ sends this point to $\left(-\tau, \tau /\left(\tau^{2}+1\right)\right)$, Equation (2.6) implies that this latter point (when $n=5$, this is the top left corner 'dark gray' point of the left region of Figure 2) lies on the curve of equation $y=\tau /(1-\tau x)$. Elementary use of partial derivatives shows that all of $\Gamma \cap\left\{x<\varepsilon_{0}\right\}$ lies exterior to $\mathcal{D}$. From $\varepsilon_{0}=-\tau^{3} /\left(1+\tau^{2}\right)$ we find

$$
\varepsilon_{n-2}=M_{1}^{-2} \cdot \varepsilon_{0}=\left(-1+\tau-\tau^{2}\right) /\left(\tau\left(2-\tau+\tau^{2}\right)\right) ;
$$

therefore, the point $(x, y)=\left(\varepsilon_{n-2}, \tau\right)$ lies on the curve $-1 / x-1 / \tau=-1+1 / \tau+\tau$. Since $\tau>1$, this point lies exterior to $\mathcal{D}$. Combining this with the fact that $P_{\infty}$ lies on the lower boundary of $\mathcal{D}$, we have that points in $\Gamma$ of $x$-coordinate greater than or equal to $1 /(1-\tau)$ all lie exterior to $\mathcal{D}$. The first result follows.

To see the optimality of the exponent $n-2$, one can certainly find $\check{Q}$ of $x$-coordinate less than $\varepsilon_{0}$ and such that all of $\check{Q}, Q=\mathcal{T}(\check{Q})$ and $\mathcal{T}^{n-3}(Q)$ lie above the curve $\theta(x, y)=\tau$. Lemma 4.1 shows that the function $\theta(x, y)$ increases along the $\mathcal{T}$-orbit of $Q$ until $y \geq-x$. For $y \geq-x$, the function $\theta(x, y)$ is decreasing along the $\mathcal{T}$-orbit. 
Thus, the $\theta$-values along the orbit from $Q$ to $\mathcal{T}^{n-3}(Q)$ increase from a value greater than $\tau$ and thereafter decrease to a value still greater than $\tau$; those all are greater than $\tau$. We conclude that all of $\left\{Q, \mathcal{T}(Q), \ldots, \mathcal{T}^{n-3}(Q)\right\} \subset \mathcal{D}$.

Proposition 4.10. For each $j \in \mathbb{N}$, there is a unique point $P_{j}=\left(x_{j}, y_{j}\right)$ in $\Gamma$ with $x_{j}$ a fixed point of $M_{1}^{n-3} W^{j} M_{2}$, and $y_{j}$ a fixed point of $\left(\begin{array}{rr}0 & -1 \\ 1 & 0\end{array}\right) M_{1}^{n-3} W^{j} M_{2}\left(\begin{array}{rr}0 & -1 \\ 1 & 0\end{array}\right)$. The point $P_{j}$ is periodic under $\mathcal{T}$ of period length $n-1$; the minimum value along the $\mathcal{T}$-orbit of $P_{j}$ of $\theta(x, y)$ is realized at $P_{j}$ itself. The limit as $j$ tends to infinity of these values equals $\tau$.

Proof. For any $x \in\left[\varepsilon_{0}, \varepsilon_{1}\right)$, we have that $f^{n-3}(x)=M_{1}^{n-3} \cdot x$. Furthermore, amongst this set of $x$, there exists a subinterval such that $f^{n-3}(x) \geq 1 /(1-\tau)$, thus for which $f^{n-2}(x)=M_{2} M_{1}^{n-3} \cdot x$. Since the image under $M_{2} M_{1}^{n-3}$ of this subinterval is $\left[-\tau, \varepsilon_{n-1}\right)$, there are smaller intervals upon which $f^{n-1}(x)=W^{j} M_{2} M_{1}^{n-3} \cdot x$. The image of each $\Delta_{-j}^{\prime}$ under $W^{j}$ is $\left[\varepsilon_{0}, 0\right)$, thus this image covers all of our initial interval. In particular, there is certainly a fixed point of $W^{j} M_{2} M_{1}^{n-3}$ there. Conjugating, we find that there is an $x_{j}$ of the type announced. One now easily verifies that there is a point $P_{j}$ fixed by $\mathcal{T}^{n-1}$, with $P_{j}=\left(x_{j}, y_{j}\right)$ and $y_{j} \in\left[0, L_{2 n-5}\right]$.

We now claim that $\lim _{j \rightarrow \infty} P_{j}=\left(1 /(1-\tau), L_{2 n-5}\right)$. Indeed, as $j$ increases, the subinterval $\Delta_{-j}^{\prime}$ tends to the left. Since $M_{2} \cdot x$ is an increasing function, the $x_{j}$ are thus decreasing, with limit value $M_{2}^{-1} \cdot(-\tau)=1 /(1-\tau)$. Similarly, the images under $\mathcal{T}$ of $\Delta_{-j}^{\prime} \times\left[0, \tau /\left(1+\tau^{2}\right)\right]$ increase in height with $j$, with heights limiting to $L_{1}$. Therefore, the $y_{j}$ limit in value to $N_{1}^{n-3} \cdot L_{1}=L_{2 n-5}$. Note that $\theta\left(1 /(1-\tau), L_{2 n-5}\right)=\tau$.

It is clear that each $y_{j}$ equals $-1 / z$ with $z$ one of the two fixed points of the hyperbolic $M_{1}^{n-3} W^{j} M_{2}$. Since $\Gamma$ contains no points on the curve $-1 / x$, it must in fact be that $y_{j}=-1 / x_{j}^{*}$, where * denotes the 'conjugate' fixed point. A direct calculation shows that $\theta\left(\mathcal{T}^{2}\left(P_{1}\right)\right)>\tau$.

For $j>1, \mathcal{T}^{2}\left(P_{j}\right)$ lies to the left and higher than $\mathcal{T}^{2}\left(P_{1}\right)$; use of partial derivatives shows that $\theta\left(\mathcal{T}^{2}\left(P_{j}\right)\right)>\theta\left(\mathcal{T}^{2}\left(P_{1}\right)\right)$. For any $j$, as in the proof of Proposition 4.9, Lemma 4.1 implies that along the $\mathcal{T}$-orbit of $\mathcal{T}^{2}\left(P_{j}\right)$ until $y=-x$, the $\theta$-values increase, thereafter they decrease until reaching $\theta\left(P_{j}\right)$. From this, the minimal value on the orbit of $P_{j}$ is taken at either $P_{j}$ or $\mathcal{T}^{2}\left(P_{j}\right)$. (Note that the lemma does not apply for an application of $W^{j}$.) But at $\mathcal{T}^{2}\left(P_{j}\right)$ the $\theta$-values are above $\tau$, and at $P_{j}$ they are below. The result follows.

Proof of Proposition 4.6 First, since $\mathcal{T}$ is ergodic, Jager's result (Proposition 2.1) shows that there are $x \in \mathbb{I}_{n}$ such that the $\mathcal{T}$-orbit of $(x, 0)$ meets the set of points $Q$ such that all of $Q, \ldots, \mathcal{T}^{n-3}(Q)$ lie in $\mathcal{D}$. Lemma 4.8 shows that such an $x$ has $n-2$ consecutive $\Theta_{m}$ values greater than $\tau$.

Second, let the point $P_{j}$ as above have coordinates $\left(x_{j}, y_{j}\right)$. Then $f^{n-2}\left(x_{j}\right)=x_{j}$ and $\mathcal{T}^{m(n-2)}\left(x_{j}, 0\right)$ converges to $P_{j}$ (with increasing $y$-values), showing the optimality in the statement of the theorem. 


\section{Transcendence results}

A basic question one can ask about a given continued fraction algorithm is if it can be used to develop criteria for determining whether or not a real number $\alpha$ is transcendental. This question was first addressed for the regular continued fractions by Maillet [23], Davenport and Roth [16], and Baker [6]. Bugeaud and Adamczewski improved upon their work in $[1,2]$. They showed that if $\xi$ is an algebraic irrational number with approximants $p_{n} / q_{n}$, then the sequence $\left\{q_{n}\right\}_{n \geq 1}$ cannot increase too quickly. Bugeaud et al. obtained similar transcendence results for the Rosen continued fractions in [11].

In this section, we prove a transcendence theorem for the Ward continued fractions in the spirit of these past results.

We recall the following theorem stated by Roth and proved by LeVeque; see [10, Ch. 4]. Note that if $\alpha$ is an algebraic number, then its naive height, denoted by $H(\alpha)$, is the largest absolute value of the coefficients of its minimal polynomial over $\mathbb{Z}$.

THeOREM 5.1 (Roth-LeVeque). Let $K$ be a number field and $\zeta$ a real algebraic number not in $K$. Then, for any $\epsilon>0$, there exists a positive constant $c(\zeta, K, \epsilon)$ such that

$$
|\zeta-\alpha|>\frac{c(\zeta, K, \epsilon)}{H(\alpha)^{2+\epsilon}}
$$

holds for every $\alpha \in K$.

Lemma 5.2. There exists a constant $c$ such that for every real number $x$ and for each $m \geq 0$,

$$
\left|x-p_{m} / q_{m}\right|<c /\left(q_{m} q_{m+1}\right) .
$$

Proof. The region $\Omega$ is bounded away from the curve $y=-1 / x$. Let $d$ be the distance from this curve to $\Omega$, and let $c=1 / d$. Then

$$
\begin{aligned}
\left|x-p_{m} / q_{m}\right| & =\frac{1}{q_{m}^{2}\left|\left(q_{m+1} / q_{m}\right)+f^{m+1}(x)\right|} \\
& =\frac{1}{q_{m} q_{m+1}\left|1+\left(q_{m} / q_{m+1}\right) f^{m+1}(x)\right|} \\
& =\frac{1}{q_{m} q_{m+1}\left|1+v_{m+1} t_{m+1}\right|} \\
& <\frac{1}{d q_{m} q_{m+1}}=\frac{c}{q_{m} q_{m+1}} .
\end{aligned}
$$

In the third equality, we used the definition $\mathcal{T}^{m+1}(x, 0)=\left(t_{m+1}, v_{m+1}\right)$, and the identity $v_{m+1}=q_{m} / q_{m+1}$.

We also have the following lemma, which appears in entirely analogous form as [11, Lemma 3.2]; their proof goes through here. 
Lemma 5.3. Let $d$ denote the field extension degree $[\mathbb{Q}(\lambda): \mathbb{Q}]$. If $\xi \notin \mathbb{Q}(\lambda)$ is a real algebraic number that is $f$-irrational, with $f$-approximants $p_{m} / q_{m}$, then there exist constants $k=k(\lambda)$ and $m_{0}=m_{0}(x)$ so that, for all $m \geq m_{0}$,

$$
H\left(p_{m} / q_{m}\right) \leq k q_{m}^{d} .
$$

It is important to note, however, that the result from [11] depends on the fact that their continued fraction algorithms arise from Fuchsian triangle groups, as do our algorithms as well. These groups have the following domination of conjugates property, by combining [28, Corollary 5] with the main result of [14].

Theorem 5.4 (Wolfart et al.). For any $M \in \mathcal{W}_{n}$ whose trace is of absolute value greater than two,

$$
|\operatorname{tr}(M)| \geq|\sigma(\operatorname{tr}(M))|
$$

where $\sigma$ is any field embedding of $Q(2 \cos (\pi / n))$.

We are now ready to give the proof for our second main theorem. We use the standard notation of $\gg$ to denote inequality with an implied constant.

Proof of Theorem 1.2. Fix $\varepsilon>0$. The Roth-LeVeque theorem implies that

$$
\left|\xi-p_{m} / q_{m}\right| \gg H\left(p_{m} / q_{m}\right)^{2+\varepsilon}, \quad n \geq 1 .
$$

And from Lemma 5.3, we have that, for $m \geq m_{0}=m_{0}(\xi)$,

$$
\left|\xi-p_{m} / q_{m}\right| \gg q_{m}^{-d(2+\varepsilon)} .
$$

Lemma 5.2 thus implies that there exists a constant $c_{1}$ such that

$$
q_{m+1}<c_{1} q_{m}^{d(2+\varepsilon)-1}
$$

for all $m \geq m_{0}$. On the other hand, for each $j<m_{0}$, there exists an $l_{j}$ such that $q_{j}<l_{j} q_{j-1}^{d(2+\epsilon)-1}$. If we let $c_{2}=\max \left\{c_{1}, l_{1}, \ldots, l_{m_{0}-1}\right\}$, then, for all $m>1$,

$$
q_{m+1}<c_{2} q_{m}^{d(2+\varepsilon)-1} .
$$

Let $a=d(2+\epsilon)-1$. Iterating this inequality once, we have that

$$
q_{m+1}<c_{2} q_{m}^{a}<c_{2}\left(c_{2} q_{m-1}^{a}\right)^{a}<\left(c_{2} q_{m-1}\right)^{a^{2}},
$$

and continuing, $q_{m+1}<\left(c_{2} q_{1}\right)^{a^{m}}$.

Since $q_{m} / q_{m+1} \leq \tau$ for all $m \geq 1, q_{m+1} \geq(1 / \tau) q_{m}$ and so by letting $c_{3}=c_{2} q_{1}$, we have that $\log q_{m}<a^{n} \log \left(c_{3}\right)$. It then follows that

$$
\limsup _{n \rightarrow \infty} \frac{\log \log q_{m}}{m}<\log (d(2+\epsilon)-1) .
$$

If we let $\varepsilon$ go to zero, we have that every algebraic number satisfies

$$
\limsup _{m \rightarrow \infty} \frac{\log \log q_{m}}{m} \leq \log (2 d-1) .
$$




\section{Acknowledgements}

We thank the Hausdorff Research Institute for Mathematics and the Mathematics Department at Oregon State University for providing stimulating environments while this research was undertaken.

\section{References}

[1] B. Adamczewski and Y. Bugeaud, 'On the complexity of algebraic numbers II: Continued fractions', Acta Math. 195 (2005), 1-20.

[2] B. Adamczewski and Y. Bugeaud, 'On the Maillet-Baker continued fractions', J. reine angew. Math. 606 (2007), 105-121.

[3] R. Adler, 'Continued fractions and Bernoulli trials', in: Ergodic Theory: A Seminar, Lecture Notes, 110 (eds. J. Moser, E. Phillips and S. Varadhan) (Courant Institute of Mathematical Sciences, New York University, New York, 1975).

[4] P. Arnoux and P. Hubert, 'Fractions continuées sur les surfaces de Veech', J. Anal. Math. 81 (2000), 35-64.

[5] P. Arnoux and T. A. Schmidt, 'Veech surfaces with nonperiodic directions in the trace field', J. Mod. Dyn. 3(4) (2009), 611-629.

[6] A. Baker, 'Continued fractions of transcendental numbers', Mathematika 9 (1962), 1-8.

[7] J. Barrionuevo, R. Burton, K. Dajani and C. Kraaikamp, 'Ergodic properties of generalized Lüroth series', Acta Arith. 74(4) (1996), 311-327.

[8] É. Borel, 'Contribution à l'analyse arithmétique du continu', J. Math. Pures Appl. 9 (1903), 329-375.

[9] W. Bosma, H. Jager and F. Wiedijk, 'Some metrical observations on the approximation by continued fractions', Indag. Math. 45(3) (1983), 281-299.

[10] Y. Bugeaud, Approximation by Algebraic Numbers, Cambridge Tracts in Mathematics, 160 (Cambridge University Press, Cambridge, 2004).

[11] Y. Bugeaud, P. Hubert and T. A. Schmidt, 'Transcendence with Rosen continued fractions', J. Eur. Math. Soc. (JEMS) 15 (2013), 39-51.

[12] R. M. Burton, C. Kraaikamp and T. A. Schmidt, 'Natural extensions for the Rosen fractions', Trans. Amer. Math. Soc. 352 (1999), 1277-1298.

[13] K. Calta and J. Smillie, 'Algebraically periodic translation surfaces', J. Mod. Dyn. 2(2) (2008), 209-248.

[14] P. Cohen and J. Wolfart, 'Modular embeddings for some nonarithmetic Fuchsian groups', Acta Arith. 56(2) (1990), 93-110.

[15] K. Dajani, C. Kraaikamp and W. Steiner, 'Metrical theory for $\alpha$-Rosen fractions', J. Eur. Math. Soc. (JEMS) 11(6) (2009), 1259-1283.

[16] H. Davenport and K. F. Roth, 'Rational approximations to algebraic numbers', Mathematika 2 (1955), 160-167.

[17] H. Jager, 'Continued fractions and ergodic theory', in: Transcendental Number and Related Topics, RIMS Kokyuroku, 599 (Kyoto University, Kyoto, 1986), pp. 55-59.

[18] R. Kenyon and J. Smillie, 'Billiards in rational-angled triangles', Comment. Math. Helv. 75 (2000), 65-108.

[19] C. Kraaikamp, 'A new class of continued fraction expansions', Acta Arith. 57(1) (1991), 1-39.

[20] C. Kraaikamp, H. Nakada and T. A. Schmidt, 'Metric and arithmetic properties of mediant-Rosen maps', Acta Arith. 137(4) (2009), 295-324.

[21] C. Kraaikamp and I. Smeets, 'Approximation results for $\alpha$-Rosen fractions', Unif. Distrib. Theory 5(2) (2010), 15-53.

[22] C. Kraaikamp, I. Smeets and T. A. Schmidt, 'Tong's spectrum for Rosen continued fractions', J. Théor. Nombres Bordeaux 19(3) (2007), 641-661. 
[23] E. Maillet, Introduction à la théorie des nombres transcendants et des propriétés arithmétiques des fonctions (Gauthier-Villars, Paris, 1906).

[24] H. Nakada, S. Ito and S. Tanaka, 'On the invariant measure for the transformations associated with some real continued-fractions', Keio Engrg. Rep. 30(13) (1977), 159-175.

[25] H. Nakada, 'On the Lenstra constant associated to the Rosen continued fractions', J. Eur. Math. Soc. (JEMS) 12(1) (2010), 55-70.

[26] V. A. Rohlin, 'Exact endomorphisms of Lebesgue spaces', Izv. Akad. Nauk SSSR Ser. Mat. 25 (1961), 499-530; Amer. Math. Soc. Transl., Series 2, 39 (1964), 1-36.

[27] D. Rosen, 'A class of continued fractions associated with certain properly discontinuous groups', Duke Math. J. 21 (1954), 549-563.

[28] P. Schmutz Schaller and J. Wolfart, 'Semi-arithmetic Fuchsian groups and modular embeddings', J. Lond. Math. Soc. 61(1) (2000), 13-24.

[29] F. Schweiger, Ergodic Theory of Fibred Systems and Metric Number Theory (Clarendon Press, Oxford, 1995).

[30] J. Smillie and C. Ulcigrai, 'Beyond Sturmian sequences: coding linear trajectories in the regular octagon', Proc. Lond. Math. Soc. 102 (2011), 291-340.

[31] J. Smillie and C. Ulcigrai, 'Geodesic flow on the Teichmüller disk of the regular octagon, cutting sequences and octagon continued fractions maps', in: Dynamical Numbers: Interplay Between Dynamical Systems and Number Theory, Contemporary Mathematics, 532 (American Mathematical Society, Providence, RI, 2010), pp. 29-65.

[32] W. A. Veech, 'Teichmuller curves in modular space, Eisenstein series, and an application to triangular billiards', Invent. Math. 97 (1989), 553-583.

[33] C. Ward, 'Calculation of Fuchsian groups associated to billiards in a rational triangle', Ergod. Theory Dynam. Sys. 18 (1998), 1019-1042.

KARIANE CALTA, Vassar College, Poughkeepsie, NY 12604-0257, USA e-mail: kacalta@vassar.edu

THOMAS A. SCHMIDT, Oregon State University, Corvallis, OR 97331, USA e-mail: toms@math.orst.edu 